\title{
Gut microbiota condition the therapeutic efficacy of trastuzumab in HER2-positive breast cancer.
}

Authors: Martina Di Modica ${ }^{1}$, Giorgio Gargari ${ }^{1}$, Viola Regondi $^{1}$, Arianna Bonizzi $^{2}$, Stefania

Arioli $^{3}$, Beatrice Belmonte ${ }^{4}$, Loris De $\mathrm{Cecco}^{5}$, Elena Fasano ${ }^{1}$, Francesca Bianchi ${ }^{1}$, Alessia

Bertolotti ${ }^{6}$, Claudio Tripodo ${ }^{4,10}$, Laura Villani ${ }^{7}$, Fabio Corsi ${ }^{2,8}$, Simone Guglielmetti ${ }^{3}$, Andrea

Balsari $^{1,9}$, Tiziana Triulzi ${ }^{1 \dagger}$ and Elda Tagliabue ${ }^{1 \dagger^{*}}$.

\section{Affiliations:}

${ }^{1}$ Molecular Targeting Unit, Dept. of Research, Fondazione IRCCS Istituto Nazionale dei Tumori, Milan, Italy.

${ }^{2}$ Dept. of Biomedical and Clinical Sciences "L. Sacco", Università degli Studi di Milano, Milan, Italy.

${ }^{3}$ Dept. of Food, Environmental and Nutritional Sciences (DeFENS), Università degli Studi di Milano, Milan, Italy.

${ }^{4}$ Tumor Immunology Unit, Dept. PROMISE, Università degli Studi di Palermo, Palermo, Italy. ${ }^{5}$ Platform of Integrated Biology, Dept. of Applied Research and Technology Development, Fondazione IRCCS Istituto Nazionale dei Tumori, Milan, Italy.

${ }^{6}$ Dept. of Pathology, Fondazione IRCSS Istituto Nazionale Tumori, Milan, Italy.

${ }^{7}$ Pathology Unit, Istituti Clinici Scientifici Maugeri IRCCS, Pavia, Italy.

${ }^{8}$ Breast Unit, Istituti Clinici Scientifici Maugeri IRCCS, Pavia, Italy.

${ }^{9}$ Dept. of Biomedical Science for Health, Università degli Studi di Milano, Milan, Italy.

${ }^{10}$ IFOM, the FIRC Institute of Molecular Oncology, Milan, Italy.

$\uparrow$ These authors contributed equally to this work.

*Corresponding author.

Running title: Gut microbiota implication in response to trastuzumab therapy.

Keywords: HER2-positive breast cancer, gut microbiota, trastuzumab.

\section{Additional information}

- Funding: This study was sponsored by Associazione Italiana Ricerca sul Cancro (Fondazione AIRC) (IG 2017 - ID.20264 project - P.I. ET). MDM was supported by a FIRC-AIRC fellowship for Italy (ID. 22304).

- Corresponding authors: Dr. Elda Tagliabue; email: elda.tagliabue@istitutotumori.mi.it; phone: +39 02 23903013; Fax: +3902 23903073

- Conflict of interest: The authors disclose no conflicts of interest.

- Words count: 5217

- Figures and tables: 6 


\section{Abstract}

Emerging evidence indicates that gut microbiota affect the response to anticancer therapies by modulating the host immune system. In this study, we investigated the impact of the gut microbiota on immune-mediated trastuzumab antitumor efficacy in preclinical models of HER2positive breast cancer (BC) and in 24 patients with primary HER2-positive BC undergoing trastuzumab-containing neoadjuvant treatment. In mice, the antitumor activity of trastuzumab was impaired by antibiotic administration or fecal microbiota transplantation from antibiotictreated donors. Modulation of the intestinal microbiota was reflected in tumors by impaired recruitment of CD4+ T cells and GZMB+ cells after trastuzumab treatment. Antibiotics caused reductions in dendritic cell (DC) activation and the release of IL12p70 upon trastuzumab treatment, a mechanism that was necessary for trastuzumab effectiveness in our model.

In patients, lower $\alpha$-diversity and lower abundance of Lachnospiraceae, Turicibacteriaceae, Bifidobacteriaceae and Prevotellaceae characterized nonresponsive patients (NR) compared to those who achieved pathological complete response $(\mathrm{R})$, similar to antibiotic-treated mice. The transfer of fecal microbiota from R and NR into mice bearing HER2-positive BC recapitulated the response to trastuzumab observed in patients. Fecal microbiota $\beta$-diversity segregated patients according to response and positively correlated with immune signature related to interferon, IL12-NO, activated CD4+ T cells and activated DC in tumors. Overall, our data reveal the direct involvement of the gut microbiota in trastuzumab efficacy, suggesting that manipulation of the gut microbiota is an optimal future strategy to achieve a therapeutic effect or to exploit its potential as a biomarker for treatment response. 


\section{Significance}

62 Evidence of gut microbiota involvement in trastuzumab efficacy represents the foundation for

63 new therapeutic strategies aimed at manipulating commensal bacteria to improve response in 64 trastuzumab-resistant patients

65 


\section{Introduction}

The aggressive biological behavior of breast carcinoma (BC) overexpressing human epidermal growth factor receptor 2 (HER2) and the consequent worse clinical outcomes of patients with these tumors (1) have largely been addressed by targeting HER2. Trastuzumab, a recombinant humanized monoclonal antibody that binds to the extracellular domain of HER2, represents the first treatment option for women with early and advanced stages of HER2-positive BC (2). Although trastuzumab substantially improves the clinical outcomes of HER2-positive BC patients, a large number of patients present or develop resistance to this treatment, underlying the need to optimize the response rate in resistant patients. Several attempts have been made to understand the reason for the lack of efficacy and to identify biomarkers that predict patients who will benefit from trastuzumab treatment (reviewed in (3)). By using the PAM50 classifier to define different tumor intrinsic subtypes within HER2-positive BC, patients with tumors classified as HER2-enriched (i.e., characterized by the high expression of ERBB2 and other genes of the 17q amplicon and low to intermediate expression of luminal genes, such as ESRI and $P G R$ ) are more likely than the others to benefit from anti-HER2 treatment (4). However, despite the high sensitivity of HER2-enriched tumors, no more than $50 \%$ of these patients respond to trastuzumab (reviewed in (3)), indicating that the effectiveness of this mAb is not determined by intrinsic tumor features only. In line with this speculation, evidence shows that the addition of anti-HER2 therapies in combination with trastuzumab (e.g., trastuzumab emtansine, pertuzumab, and lapatinib) remains ineffective in many resistant patients (5).

The importance of the host immune system in the mechanism of action of trastuzumab has become increasingly clear (reviewed in $(6 ; 7)$ ), indicating that trastuzumab not only inhibits HER2-triggered signal transduction but also has immunomodulatory properties. Patients with 
highly infiltrated tumors or tumors expressing a particular subset of immune system genes have a

lower risk of relapse than others upon trastuzumab treatment (7). However, even considering tumor and/or immune microenvironment characteristics, the prediction of trastuzumab benefit did not result in sufficient accuracy for clinical practice $(3 ; 8)$, indicating that host-related features might add missing clues to identify sensitive/resistant patients.

The gut microbiota has been described as one of the major environmental factors that is able to regulate the development and maintenance of the immune system. Recently, studies in preclinical models (9-13) and patient cohorts $(12 ; 14-18)$ have clearly shown the causal role of commensal communities in the efficacy of both chemotherapy and immunotherapy through the modulation of host immunity.

Based on the relevance of the patient immune system to the therapeutic effect of trastuzumab and the importance of gut commensal bacteria in host immune system maintenance, in this study, we investigated, in experimental models and HER2-positive BC patients, the role of gut microbiota as an extrinsic tumor feature contributing to the response to trastuzumab through regulation of the pre-existing or trastuzumab-conditioned tumor immune microenvironment. 


\section{Materials and Methods}

\section{Antibiotic treatment and in vivo experiments}

Female FVB/Ncrl mice (four weeks old) were purchased from Charles River Laboratories (Calco, Italy; cat no. CRL:207; RRID:IMSR_CRL:207). The mice were treated with a single antibiotic (vancomycin or streptomycin dissolved in drinking water, $200 \mathrm{mg} / \mathrm{L}$ ) for the entire duration of the experiment and water was used as control (NoA). These antibiotics were seleted because poorly absorbed in the intestine and for their different mechanisms of action with vancomycin mainly directed against Gram-positive bacteria (19) and streptomycin as a broad spectrum protein synsthesis inhibitor (20). After four weeks of antibiotic treatment, $1 \times 10^{6}$ human HER2-positive MI6 murine mammary carcinoma cells were injected into the mouse mammary fat pad. When tumors reached a palpable volume (3-4 $\mathrm{mm}$ in diameter), the mice were randomized into two groups and treated biweekly with intraperitoneal (i.p.) injections of trastuzumab $(5 \mathrm{mg} / \mathrm{kg}$ body weight), or saline $(\mathrm{NaCl} 0.9 \%)$ as control, for the duration of the entire experiment. The tumors were measured by caliper, and the volume was calculated as 0.5 $\times \mathrm{d} 1^{2} \times \mathrm{d} 2$, where $\mathrm{d} 1$ and $\mathrm{d} 2$ are the smaller and larger diameters, respectively. For the depletion experiments the following InVivoMAb antibodies (BioXcell, Lebanon, NH, USA) were used: rat IgG2b isotype control, clone LTF-2 (400 $\mu$ g i.p. twice a week) (cat no. BE0090; RRID:AB_1107780); anti-mouse CD4, clone GK1.5 (400 $\mu \mathrm{g}$ i.p. twice a week) (cat no. BE0003-1; RRID:AB_1107636); anti-mouse IL12p70, clone R2-9A5 (1 mg the day before the first trastuzumab injection and then $500 \mu \mathrm{g}$ i.p twice a week (21)) (cat no. BE0233; RRID:AB_2687715). Recombinant mouse IL12p70 (rIL12p70) (Biolegend, cat no. 577006) was administered to mice under vancomycin, starting the day before trastuzumab administration (500 ng i.p three times a week) (adapted from (22)). Experimental protocols 
132 used for animal studies were approved by the institutional review board and by the Italian

133 Ministry of Health.

134 Detailed protocols for experiments carried out in FVB $\triangle 16 \mathrm{HER} 2$ transgenic mice can be found

135 in supplementary materials and methods.

136

137

138

139

140

141

142

143

144

145

146

147

148

149

150

151

152

153

\section{Patient cohort}

In this study, we analyzed 24 consecutive patients who received neoadjuvant trastuzumab-based chemotherapy between 2017 and 2019 at the Istituti Clinici Scientifici Maugeri of Pavia. Twenty patients received 4 cycles of AC (adriamycin plus cyclophosphamide), followed by four to six cycles of $\mathrm{TH}$ (taxane and trastuzumab) as therapy, while four patients received taxane plus trastuzumab for six cycles since the beginning. Pathological complete response (pCR) was defined as no residual invasive tumor in the complete resected breast specimen. Table 1 lists the characteristics of patients and diseases according to the response. Fecal samples from patients were collected before the beginning of $\mathrm{TH}$. The biospecimens consisted of leftover material from samples that had been collected during standard biopsy surgical and medical procedures at the Istituti Clinici Scientifici Maugeri - Breast Unit. Samples were donated by patients to the Institutional BioBank for research purposes, and aliquots were designated for this study after approval by the institutional review board and by an independent ethical committee of the Istituti Clinici Scientifici Maugeri and the Fondazione IRCCS Istituto Nazionale dei Tumori. All procedures were performed in accordance with the Declaration of Helsinki and all subjects signed a written informed consent for the study. Additional information can be found in supplementary materials and methods.

\section{Fecal microbial transplantation (FMT) experiment}


154 The intestinal flora of four-week-old FVB mice was depleted by feeding the animals for 28 days

155

156

157

158

159

160

161

162

163

164

165

166

167

168

169

170

171

172

173

174

175

176 with an antibiotic cocktail $(\mathrm{ABX})$ (neomycin, ampicillin, metronidazole $1 \mathrm{~g} / \mathrm{L}$ and vancomycin $500 \mathrm{mg} / \mathrm{L})$, as described $(23 ; 24)$. The feces of antibiotic-treated donor mice were homogenized in prereduced 1X PBS at a concentration of $130-150 \mathrm{mg} / \mathrm{ml}$. Fecal suspensions $(200 \mu \mathrm{l})$ were delivered to mice via oral gavage twice a week for 2 weeks and then once a week until the end of the experiment. Trastuzumab treatment started when the tumor reached a palpable volume as described above. Further details on FMT with patients stool sample are reported in the supplementary materials and methods.

Immune characterization and plasma cytokines quantification Detailed protocols can be found in Supplementary Materials and Methods. Supplementary Table 1 lists the antibodies used.

\section{Fecal sample analysis}

Metagenomic DNA was extracted from $250 \mathrm{mg}$ (or from one pellet in mice) of stool using a PowerLyzer PowerSoil DNA isolation kit (Qiagen, cat no. 12855-100, Hilden, Germany). Starting from $12.5 \mathrm{ng}$ of total DNA, the bacterial community structure was determined by the sequencing of the variable region 3 and 4 (V3 and V4) of the 16S rRNA gene on the MiSeq Illumina technology platform at the Center for Life Nanosciences, Italian Institute of Technology (Rome, Italy). The sequence reads were then analyzed using the bioinformatics pipeline Quantitative Insights Into Microbial Ecology (QIIME) version 1.9.1 (25). Bacterial abundances in each fecal sample were reported at the taxonomic levels of phylum, order, family and genus.

\section{Gene expression and bioinformatics analysis}

For patient's study, RNA was extracted from FFPE BC core biopsies using the miRNAeasy FFPE kit (Qiagen, cat no. 217504). A total of 13 tumor core biopsies collected at diagnosis 
177 before neoadjuvant treatment were available out of 24 patients. Gene expression profile was

178 carried out using Clariom ${ }^{\mathrm{TM}} \mathrm{S}$ Pico Assay (Thermo Fisher Scientific), a detailed protocol for the

179 gene expression profile can be found in supplementary materials and methods. The data were

180 deposited into the Gene Expression Omnibus (GEO; RRID:SCR_005012) repository (accession

181 number GSE149283). The research-based PAM50 subtype predictor was applied using the

182 publicly available algorithm as described after merging the dataset with 50 consecutive BC cases

183 profiled on the same platform and performing median centering of the PAM50 genes (26).

184 For ileum and colon samples, RNA was extracted from frozen samples using miRNeasy mini Kit

185 (Qiagen, cat no. 217004). Gene expression was performed as described above, the data were deposited into the GEO repository (accession number GSE149712).

187 Functional annotation clustering of differentially expressed genes (DEGs) between NoA and vancomycin was performed by DAVID Bioinformatics Resources v6.8 (RRID: SCR_001881)

189 (27). Gene-set enrichment analyses (GSEA; RRID:SCR_003199) in the intestines were 190 performed by GSEA v4.0.3 (28) using a selection of immune pathways from GO biological

191 processes and KEGG pathways gene set. In patients' tumors, GSEA analysis was performed in 192 continuous based on PC1 values of patient's $\beta$-diversity analysis using a previously described 193 cancer-related geneset (29). Pearson metric for ranking genes was used. Gene set permutation 194 type was applied 1000 times and gene set enrichment was considered significant at FDR $<10 \%$.

195 Immune metagenes were determined per Rody et al. (30). The average log-transformed 196 expression of the genes that belonged to each metagene was calculated. Single-sample GSEA 197 (ssGSEA) was performed with previously published immune signatures (31).

$198 \quad$ Statistical analyses 
Analyses were performed using GraphPad Prism 5.0 (GraphPad Software; RRID:SCR_002798).

Differences between the groups were determined using a two-tailed unpaired t-test. The association among categorical variables was tested by Fisher's exact test and correlation between continous variables were examined by Spearman correlation analysis. Differences were considered significant at $\mathrm{p}<0.05$. The software $\mathrm{R}$ version 3.4 .2 was used for the statistics concerning the microbiota analysis. The Linear discriminant analysis Effect Size (LEfSe; RRID:SCR_014609) algorithm was used to discover taxa differences between groups (32). Specifically, the algorithm uses the nonparametric factorial Kruskal-Wallis sum-rank test associated with a p-value correction test to detect features with significant differential abundance with respect to the group of interest. Statistical significance was set at $p \leq 0.05$, and mean differences with $0.05<\mathrm{p} \leq 0.10$ were accepted as trends. The ecological diversity ( $\alpha$ and $\beta$ ) was calculated by QIIME software version 1.9.1 (RRID:SCR_008249). Concerning the $\alpha$-diversity

211 (intrasample diversity), we used three different indexes: Chao1, Shannon and Simpson. The first 212 index examines the richness of different bacteria present in each sample. The second and the

213 third indexes also evaluate importance at the evenness and richness levels. The $\beta$-diversity, 214 described as intersample diversity, was measured using the UniFrac distance metric (33). This distance was used because it incorporates information on the relative relatedness of community

216 members by incorporating phylogenetic distances between the observed bacteria, and principal 217 coordinate analyses were performed to visually compare the microbiota of the different treatment 218 groups considering the bacterial phylogenetic distances. In the analysis of the patient $\beta$-diversity, 219 an analysis of similarities (ANOSIM) (34) was performed to determine the significance of the 220 dissimilarities observed between responsive (R) and non responsive (NR) patients. LEfSe was 221 performed to identify differentially abundant taxa in groups of treatment or R and NR patients. 


\section{Results}

\section{Antibiotic administration reduces trastuzumab therapeutic activity in preclinical models}

To address the role of commensal bacteria in the therapeutic benefit of HER2 inhibition, the antitumor activity of trastuzumab was investigated in conventional (no antibiotic, NoA) FVB mice bearing MI6 cells, and in mice whose intestinal microflora was altered by the use of vancomycin or streptomycin, two broad-spectrum antibiotics that are poorly absorbed in the intestine. The anti-HER2 mAb showed reduced antitumor efficacy in mice under antibiotic regimens (Fig. 1A; Supplementary Fig. S1A). No consequences on HER2 expression and phosphorylation (Supplementary Fig. S1B and C) or on trastuzumab distribution in tumors (Supplementary Fig. S1D) were observed following antibiotic treatment, except for a slight decrease in tumor growth in vancomycin-treated mice, ruling out possible antibiotic-induced tumor changes that led to the inefficacy of trastuzumab. The causal contribution of the gut microbiota to trastuzumab efficacy was investigated in mice whose intestinal microbiota was depleted by an antibiotic cocktail $(\mathrm{ABX})$, and then the gut was recolonized through a fecal microbiota transplant (FMT) by using a fecal suspension obtained from vancomycin or NoA donor mice (Fig. 1B; Supplementary Fig. S2A-S2C). The inhibition of tumor growth observed after trastuzumab treatment was more effective in mice transplanted with stool from NoA animals (FMT-NoA) than in mice receiving feces from vancomycin-treated donors (FMTvancomycin).

The impact of vancomycin administration on the benefit of trastuzumab therapy was also investigated in FVB- $\triangle 16$ HER2 transgenic female mice (35), a model of spontaneous mammary carcinoma. Under vancomycin treatment, the mice did not benefit from trastuzumab 
administration compared to the NoA group (Supplementary Fig. S3A). No impact of vancomycin on tumor onset or multiplicity was observed (Supplementary Fig. S3B and S3C).

\section{Vancomycin and streptomycin significantly alter the gut microbiota composition}

The bacterial community structure in the gut of antibiotic-treated mice was analyzed by $16 \mathrm{~S}$ rRNA gene profiling. Both antibiotics significantly reduced bacterial taxonomic richness, with vancomycin having a stronger impact on the microbiota than streptomycin, as reflected by the Chao1 and Shannon $\alpha$-diversity indexes (Fig. 2A) and the $\beta$-diversity PCoA plot (Fig. 2B), where the microbiota of the antibiotic-treated mice was segregated from that of the NoA mice (Fig. 2B). Consistently, analysis of microbial $\beta$-diversity in the feces of transplanted mice collected at the end of the experiment showed that although the FMT-vancomycin mice did not thoroughly recapitulate the bacterial community of donor mice, they clustered separately from the FMT-NoA mice (Supplementary Fig. S2C).

Antibiotic administration resulted in a substantial decrease in the relative abundance of bacteria belonging to the Actinobacteria and Firmicutes phyla. Vancomycin treatment also caused a substantial loss of Bacteroidetes, with a concomitant increase in the relative abundance of the phyla Proteobacteria and Verrucomicrobia. Within the phylum Firmicutes, both antibiotics reduced numerous taxonomic units belonging to the order Clostridiales, particularly to the family Lachnospiraceae (Supplementary Fig. S4A). To further explore these data, LEfSe analysis was performed, and the taxonomic families Lachnospiraceae, Turicibacteriaceae, Coriobacteriaceae and Prevotellaceae were less abundant in antibiotic-treated mice (Fig. 2C and D; Supplementary Fig. S4C and S4D). These bacteria are producers of short-chain fatty acids (SCFAs), and their low abundance in the gut of vancomycin- or streptomycin-treated mice negatively impacted the fecal levels of butyrate, propionate and acetate (Supplementary Fig. S5). 
The immune infiltrate of tumors collected at the end of the experiments with antibiotics was analyzed by immunohistochemistry (IHC) and flow cytometry (Fig. 3). Antibiotic treatment increased the density of CD45+ positive cells within the tumor masses (Fig. 3A; Supplementary Fig. S6A and S6B) along with an overall reduction in the number of CD3+ tumor-infiltrating lymphocytes (Fig. 3B; Supplementary Fig. S6A and S6C) and an increase in Gr-1+ myeloid cells (Fig. 3C; Supplementary Fig. S6A and S6D), as highlighted by IHC. Regarding the immune populations relevant for trastuzumab antitumor activity (NK cells, CD4+ $\mathrm{T}$ cells and CD8+ T cells) (36;37), we found that granzyme B (GZMB)-expressing cells were increased upon trastuzumab treatment in control animals, while the recruitment of cytotoxic effectors was impaired in vancomycin- or streptomycin-treated mice (Fig. 3D; Supplementary Fig. S6A). CD4+ $\mathrm{T}$ cells were mainly relocalized within tumor cell foci in NoA animals, whereas no redistribution was found in antibiotic-treated mice (Fig. 3E; Supplementary Fig. S6A) upon trastuzumab administration. Of note, a very small number of CD8+ $\mathrm{T}$ cells infiltrated MI6 tumors, and they were mainly localized within the stromal compartment (Supplementary Fig. S6A and S6E), suggesting that GZMB+ cells in our model are mainly represented by cytotoxic NK cells (Supplementary Fig. S6A and S6F). This speculation was supported by flow cytometry 284 analysis of an independent experiment in which an increase in CD49b+NKp46+ NK cells (Fig. 3F), but not CD8+ T cells (Supplementary Fig. S7A and S7B) or CD4+ T cells (Fig. 3G), was 286 found in tumors upon trastuzumab administration. Notably, antibiotic treatment impaired the 287 basal activation status, evaluated as CD69 expression, in NK cells and tumor-infiltrating T lymphocytes (Fig. 3F and G; Supplementary Fig. S7A and S7B). 
The analysis of tumors from FMT mice showed a similar increase in myeloid infiltrate in the tumors of FMT-vancomycin mice (Supplementary Fig. S8A), and the number of intratumor CD4+ $\mathrm{T}$ and GZMB-expressing cells increased upon treatment with trastuzumab in only the

292 FMT-NoA group (Supplementary Fig. S8B and S8C).

Gut microbiota modification affects intestinal mucosal immunity and systemic cytokine

294 circulation

The gene expression profile was analyzed in the ileum and colon of mice treated with vancomycin, and while significant changes were observed in both intestinal tracts, the ileum was the most affected by vancomycin administration, with a larger number of differentially expressed genes $(\mathrm{DEG})(\mathrm{FDR}<0.1)$ than the colon (Supplementary Fig. S9A and S9B). Functional analysis of the DEG list from the ileum revealed enrichment of pathways related to antigen presentation via MHC class II, response to IFN $\gamma$ and IgGA production in NoA animals (Fig. 4A and Table S2), as confirmed by the impaired response to interferons (IFN $\gamma$ and type I IFN) and by the reduced antigen presentation machinery highlighted by GSEA analysis in vancomycin-treated mice (Table S3). No statistically significant pathways emerged from the DEG list in colon samples, while comparing NoA and vancomycin by GSEA revealed enrichment of pathways related to the positive regulation of macrophage and myeloid cytokine production in the colon of NoA animals (Table S4).

To link changes that occurred in the gut to systemic immune tone, a panel of 26 cytokines and chemokines was measured in the plasma of NoA and vancomycin-treated mice (Fig. 4B). Most of the cytokines were below the detection limit, while a trend of higher CCL11 and CCL7 or lower CCL5 and IL12p70 levels in the vancomycin group than in the NoA group was found. Interestingly, IL12p70 significantly increased upon trastuzumab administration in only NoA 
312 mice, which likely reflects the activation status (CD86 expression) of DCs found in the tumor-

313 draining lymph nodes (dLNs) (Supplementary Fig. S9C and S9D). The functional role of

314 IL12p70 in our model with regard to trastuzumab efficacy was investigated in NoA mice: the

315 neutralization of IL12p70 through an anti-IL12p70 mAb impaired the antitumor activity of

316 trastuzumab, with a parallel significant decrease in NK cells recruitment within the tumor (Fig.

317 4C and D), while no impact on their activation, or CD4+ T cells, was observed (Supplementary

318 Fig. S10A and S10B). Conversely, the administration of recombinant IL12p70 (rIL-12p70) to

319 mice under vancomycin treatment restored the efficacy of trastuzumab (Fig. 4E), increasing NK

320 cells recruitment and basal activation (Fig. 4F; Supplementary Fig. S10C) in tumors. A similar

321 increase was observed for CD4+ T cells (Supplementary Fig. S10D). Unexpectedly, when CD4+

322 T cells were depleted in NoA mice before trastuzumab treatment, a slight improvement in anti-

323 HER2 mAb efficacy was observed (Supplementary Fig. S10E), suggesting that NK cells play a

324 major role in our model and that the microbiota-DC activation axis influences trastuzumab

325 efficacy by regulating NK cell activation and recruitment through an IL12p70-dependent

326 mechanism.

327 The gut microbiota contributes to trastuzumab benefit in HER2-positive BC patients

328 To translate our findings to the clinical setting, we analyzed 24 consecutive primary HER2-

329 positive BC patients who were treated with neoadjuvant therapy containing trastuzumab. Sixteen

330 patients experienced pCR and were considered responsive $(\mathrm{R})$, while eight presented residual

331 disease at surgery (NR) (Table 1). To investigate the composition of the commensal microbiota,

332 DNA was extracted from stool samples collected before the beginning of trastuzumab treatment,

333 and the 16S rRNA gene was profiled. Metagenomic analysis was successfully carried out for 23

334 samples $(\mathrm{R}, \mathrm{n}=16$ and $\mathrm{NR}, \mathrm{n}=7)$. $\alpha$-Diversity analysis with the Chao1, Shannon and Simpson 
indexes revealed significantly higher diversity in R patients than in NR patients (Fig. 5A). A clustering effect between $\mathrm{R}$ and NR patients (ANOSIM $p=0.029$ ) (Fig. 5B) was shown by $\beta$ diversity analysis; the higher PC1 values were, the larger the number of R patients. PAM50 molecular classification was applied to the GEP of tumor core biopsies (i.e., represented in the PCoA plot as HER2-enriched ( $\square)$ or non HER2-enriched $(\Delta)$ ), showing that microbiota clustering was independent of the tumor molecular subtypes (Fig. 5B).

Differentially abundant bacterial taxa between R and NR were then investigated by LEfSe. Patients who achieved pCR were characterized by a microbiota enriched in bacteria from the Clostridiales (i.e., Lachnospiraceae), Bifidobacteriaceae, Turicibacteraceae and Bacteroidales (i.e., Prevotellaceae family) taxonomic orders, while the phylum Bacteroidetes (such as the class Bacteroidia) was more abundant in NR patients (Fig. 5C). The link between patients' gut microbiota and the response to trastuzumab was evaluated by FMT of fecal material from R $(n=5)$ and NR $(n=4)$ patients into recipient mice (Fig. 5D). Notably, FMT-R mice benefitted the most from anti-HER2 treatment (Fig. 5D; Supplementary Fig. S11A and S11B), recapitulating the response observed in donor patients and strengthening the idea of direct involvement of commensal bacteria in trastuzumab effectiveness.

Differences in the tumor immune infiltrate between $\mathrm{R}$ and NR were investigated by applying immune signatures as a surrogate for immune cell infiltration to the GEP of tumor core biopsies. No significant differences were observed when comparing the two groups; however, we found that signatures significantly correlated with $\mathrm{PC} 1$ values, i.e., the main descriptor of $\beta$-diversity separated R from NR according to the microbiota. In particular, a positive correlation between gut microbiota composition and STAT1 metagene was found, and a trend was also observed for the MHCII metagene (Supplementary Fig. S11C). Moreover, immune signatures related to 
358 lymphocyte infiltration, B cells, activated CD4 T cells and activated DCs were found to be 359 significantly positively correlated with PC1 (Supplementary Fig. S11C). Similarly, immune 360 pathways related to interferon and IL12-NO were enriched in patients with higher PC1 values 361 (R), while non immune pathways such as electron transport chain, oxidative phosphorylation and 362 luminal genes were enriched in patients with lower PC1 values (mainly NR) (FDR<10\%) (Fig. 363 5E). IL12 is one of the leading genes in these pathways, and although no differential IL12 364 expression was found between R and NR, its levels correlate with intestinal $\beta$-diversity (Fig. 5F), 365 emerging as a possible link between gut microbiota composition, tumor immune infiltration and 366 trastuzumab efficacy in patients. 


\section{Discussion}

371 This study provides evidence that the host gut microbiota composition plays a role in trastuzumab efficacy. Vancomycin or streptomycin administration resulted in the complete abrogation of tumor growth inhibition by trastuzumab. Despite the different mechanisms of action of the two antibiotics, we found a similar decrease in bacteria belonging to the taxonomic phyla Clostridiales (i.e., Lachnospiraceae), Actinobacteria (i.e., the Coriobacteriaceae taxonomic family), Turicibacteriaceae, and Bacteroidetes (specifically, the family Prevotellaceae) upon treatment with both antibiotics, raising the possibility that certain bacteria, rather than the general diversity of the gut microbial community, may be of particular relevance for trastuzumab therapeutic activity. The reduction in taxa belonging to the order Clostridiales, specifically to the Lachnospiraceae family, can explain the reduction of butyrate, propionate and acetate observed in antibiotic-treated mice; these compounds are usually exploited as a source of energy by intestinal epithelial cells (IECs) to favor the maintenance of barrier stability. The reductions in their concentrations not only reflect the disruption of the intestinal microflora that occurs upon antibiotic administration but also result in the modulation of mucosal immunity (38).

The existence of a gut microbiota/immune-mediated trastuzumab activity axis was strongly 387 supported by the lower basal activation of tumor-infiltrating NK and CD4+ T cells as well as by a significant decrease in the recruitment of CD4+ T lymphocytes and GZMB+ cells (mainly NK 389 cells) within the tumor upon trastuzumab treatment in antibiotic-treated mice. These 390 modifications of the tumor immune microenvironment induced by the alteration of the gut 391 microbiota, rather than its impact on tumor growth (39), are likely to be the reason for a reduced response to trastuzumab treatment, as tumor proliferation has never been associated with the 
efficacy of anti-HER2 monotherapy in patients (40;41). Remarkably, by transferring fecal material from NoA and vancomycin-treated mice into recipient mice, the response to trastuzumab and the tumor immune infiltrate scenario were recapitulated in FMT mice, revealing a cause-effect link between the gut microbiota and immune-mediated trastuzumab activity. microbiota depletion by antibiotics or germ-free conditions (42), pathways such as antigen presentation and processing were diminished in the ileum of vancomycin-treated animals. Moreover, the enrichment in NoA animals of pathways associated with the inflammatory response and type I interferons is in line with the capability of commensal bacteria to instruct mononuclear phagocytes, such as DCs, to maintain a proper tone at steady state (43), which renders them ready for prompt activation upon stimulation. While further studies are needed to better understand the mechanisms through which gut bacteria sustain a DC tone favorable for trastuzumab efficacy, we found an increase in circulating IL12p70 upon trastuzumab treatment in only NoA-responsive mice, likely reflecting DC activation in the lymph nodes, supporting the causal involvement of this cytokine in the gut microbiota-mediated regulation of trastuzumab antitumor activity, as previously shown in the context of CTLA-4 and PD-1 blockade $(11 ; 12)$. Similar to vancomycin, no modulation of IL12p70 upon trastuzumab was observed in 410 streptomycin-treated mice, strengthening evidence for the role of this cytokine. IL12p70 is a Th1 411 cytokine released by microbiota sensing, activated APCs to induce the effector functions of T 412 and NK cells (44), and it has been previously described to have an adjuvant effect on 413 trastuzumab activity in mice (22). The antithetical modulation of NK cells tumor infiltration by 414 an anti-IL12p70-depleting mAb and rIL12p70 strongly supports the key role of IL12p70 in 415 mediating the gut microbiota regulation of NK cells expansion and activity in trastuzumab- 
coated cells in our in vivo model. The administration of rIL12p70 modulated CD4+ T cells in vancomycin-treated mice, and although CD4+ T cells were dispensable for trastuzumab activity in our model, this modulation highlights an alternative way through which gut bacteria may affect the trastuzumab response, increasing CD4+ priming in the ileum (42), and effector activity via IL12 secreted by DCs (12), particularly in patients where CD4+ T cells have been reported to be relevant for trastuzumab activity $(37 ; 45 ; 46)$. Dysregulation of $\mathrm{T}$ cell activity may also occur in the colon as a consequence of antibiotic-induced disruption of macrophage homeostasis (47), as we observed in the colon of vancomycin-treated mice.

The clinical relevance of these findings is supported by the results obtained in HER2-positive BC patients treated with trastuzumab-containing therapy in the neoadjuvant setting. Similar to vancomycin-treated mice, compared to that of R patients, the gut microbiota of NR patients was characterized by lower $\alpha$-diversity and higher abundance of Bacteroides. In particular, as occurred in mice under antibiotic treatment, low abundance of members of the Lachnospiraceae, Prevotellaceae, Actinobacteria (i.e., Bifidobacteriaceae), Turicibacteriaceae and Desulfovibrio taxonomic families emerged in the gut microbiota of NR women, highlighting the relevance of these bacteria for trastuzumab benefit and encouraging further studies to understand whether they have a direct role in the antibody mechanism of action. Although 16S rRNA gene sequencing did not allow us to identify bacteria to the species level, similarities with published studies on the response to immunotherapy were observed, as bacteria belonging to Lachnospiraceae (order Clostridiales) and Bifidobacteriaceae are more abundant in patients responsive to anti-PD1 treatment, while Bacteroidales characterized the microbiota of NR patients, as found in (15). 
Microbial $\beta$-diversity segregated patients according to the response to treatment, and the absence of an association between microbiota and HER2-enriched tumor intrinsic subtype suggests that the patients' gut microbial ecosystem contributes to therapy benefit independently of tumor intrinsic subtype. This evidence might explain why not all BCs scored as HER2-enriched benefit from treatment, underlining how the gut microbiota of HER2-positive BC patients can add relevant information for the prediction of trastuzumab efficacy independent of tumor molecular characteristics. In addition to representing a potential predictive biomarker, our data show that the gut microbiota plays an active role in trastuzumab activity, as demonstrated by transferring fecal material from R and NR donors into "avatar mice" that recapitulated the response observed in the clinical setting.

Notably, the correlation of microbial $\beta$-diversity with immune pathways relevant for immune cell activation and trastuzumab activity (i.e., interferon; IL12-NO; STAT1 metagene) (36;41) found in basal tumor biopsies supports the influence of the gut microbiota in shaping pre-existing tumor immune infiltrate. In addition, correlations with lymphocyte infiltration (i.e., activated CD4+ T cells) and activated DCs suggest the involvement of the microbiota-sensing APCs/IL12 axis in HER2-positive BC patients. Based on the marked differences that emerged in the tumor immune infiltrate upon trastuzumab treatment in our in vivo experiments, and based on the immunological changes observed in patients according to response to treatment after a single dose of trastuzumab $(41 ; 48)$, it is likely that the evaluation of local and systemic immune modulation upon brief exposure to trastuzumab would highlight a stronger association with gut microbiota characteristics in patients.

The direct involvement of the gut microbiota in trastuzumab activity sets the starting point for the exciting possibility of manipulating gut bacteria to improve the success of anti-HER2 
461 treatment. In this context, the low abundance of Clostridiales commonly found in antibiotic-

462 treated mice and in NR patients suggests that a dietary intervention that increases the amount of

463 fiber or is supplemented with favorable prebiotics may boost immune-mediated trastuzumab

464 activity. This intervention has also been under investigation for immune checkpoint inhibitor

465 agents (49) based on a similar reduction in bacteria associated with fiber consumption found in

466 NR patients (15). Further studies and larger clinical cohorts are needed to understand whether

467 bacteria specifically related to the trastuzumab response exist or whether Clostridiales and

468 Bacteroidales, which were found to be enriched in the gut of patients responsive and

469 unresponsive to immune checkpoint blockade, respectively(15), can also be considered overall

470 'good' and 'poor' bacteria for trastuzumab activity. In an era in which dual anti-HER2

471 combinations are becoming a common clinical practice (50), we believe that knowing the

472 favorable gut microbiota composition for trastuzumab efficacy could impact de-escalating

473 strategies in terms of single agent versus dual blockade, minimizing overtreatment in patients

474 who would benefit from single-agent trastuzumab treatment. 
Acknowledgments: The authors thank Ghirelli C. for technical support. Acknowledgment to the Microscopy and the Genomic core Facilities of the Fondazione IRCCS Istituto Nazionale dei Tumori, Milan. We thank Dr. Di Nicola M. and Dr. Cappelletti V. of the Fondazione IRCCS Istituto Nazionale dei Tumori for providing 50 consecutive BC cases profiled using the human Affymetrix Clariom S Pico assay to perform median centering of the PAM50 genes. The research leading to these results has received funding from AIRC under IG 2017 - ID. 20264 project - P.I. E. Tagliabue. M. Di Modica was supported by a FIRC-AIRC fellowship for Italy (ID. 22304).

\section{Author contributions:}

MDM, ABa, ET, and TT conceived and designed the study. ABo and FC collected human materials and data. MDM, VR, SA, BB, EF, FB performed the experiments. BB, $\mathrm{ABe}$ and CT analyzed and interpreted IHC data. LDC, performed and analyzed GEP. MDM, GG, SG, ET, and TT analyzed and interpreted the data. MDM, ET, and TT wrote the manuscript. All authors critically revised the manuscript.

Data and materials availability: All data related to this study are present in the paper or the Supplementary Materials. Gene expression data of the tumor core biopsies and mice intestine are available in the GEO repository (GSE149283 and GSE149712). 


\section{References List}

1. Slamon DJ, Clark GM, Wong SG, Levin WJ, Ullrich A, McGuire WL. Human breast cancer: correlation of relapse and survival with amplification of the HER-2/neu oncogene. Science 1987;235:177-82.

2. Maximiano S, Magalhaes P, Guerreiro MP, Morgado M. Trastuzumab in the Treatment of Breast Cancer. BioDrugs 2016;30:75-86.

3. Triulzi T, Bianchi GV, Tagliabue E. Predictive biomarkers in the treatment of HER2positive breast cancer: an ongoing challenge. Future Oncol 2016;12:1413-28.

4. Prat A, Carey LA, Adamo B, Vidal M, Tabernero J, Cortes J, et al. Molecular features and survival outcomes of the intrinsic subtypes within HER2-positive breast cancer. $\mathrm{J}$ Natl Cancer Inst 2014;106:dju152.

5. Veeraraghavan J, De Angelis C, Reis-Filho JS, Pascual T, Prat A, Rimawi MF, et al. Deescalation of treatment in HER2-positive breast cancer: Determinants of response and mechanisms of resistance. Breast 2017;34:S19-S26.

6. Bianchini G, Gianni L. The immune system and response to HER2-targeted treatment in breast cancer. Lancet Oncol 2014;15:e58-e68.

7. Di Modica M, Tagliabue E, Triulzi T. Predicting the efficacy of HER2-targeted therapies: a look at the host. Dis Markers 2017;2017:7849108.

8. Dieci MV, Prat A, Tagliafico E, Pare L, Ficarra G, Bisagni G, et al. Integrated evaluation of PAM50 subtypes and immune modulation of pCR in HER2-positive breast cancer patients treated with chemotherapy and HER2-targeted agents in the CherLOB trial. Ann Oncol 2016;27:1867-73.

9. Iida N, Dzutsev A, Stewart CA, Smith L, Bouladoux N, Weingarten RA, et al. Commensal bacteria control cancer response to therapy by modulating the tumor microenvironment. Science 2013;342:967-70 doi: 10.1126/science.1240527.

10. Viaud S, Saccheri F, Mignot G, Yamazaki T, Daillere R, Hannani D, et al. The intestinal microbiota modulates the anticancer immune effects of cyclophosphamide. Science 2013;342:971-6 doi: 10.1126/science.1240537.

11. Vetizou M, Pitt JM, Daillere R, Lepage P, Waldschmitt N, Flament C, et al. Anticancer immunotherapy by CTLA-4 blockade relies on the gut microbiota. Science 2015;350:1079-84 doi: 10.1126/science.aad1329.

12. Routy B, Le Chatelier E, Derosa L, Duong CPM, Alou MT, Daillere R, et al. Gut microbiome influences efficacy of PD-1-based immunotherapy against epithelial tumors. Science 2018;359:91-7. 
13. Sivan A, Corrales L, Hubert N, Williams JB, quino-Michaels K, Earley ZM, et al. Commensal Bifidobacterium promotes antitumor immunity and facilitates anti-PD-L1 efficacy. Science 2015;350:1084-9 doi: 10.1126/science.aac4255.

14. Matson V, Fessler J, Bao R, Chongsuwat T, Zha Y, Alegre ML, et al. The commensal microbiome is associated with anti-PD-1 efficacy in metastatic melanoma patients. Science 2018;359:104-8.

15. Gopalakrishnan V, Spencer CN, Nezi L, Reuben A, Andrews MC, Karpinets TV, et al. Gut microbiome modulates response to anti-PD-1 immunotherapy in melanoma patients. Science 2018;359:97-103.

16. Chaput N, Lepage P, Coutzac C, Soularue E, Le Roux K, Monot C, et al. Baseline gut microbiota predicts clinical response and colitis in metastatic melanoma patients treated with ipilimumab. Ann Oncol 2017;28:1368-79.

17. Derosa L, Hellmann MD, Spaziano M, Halpenny D, Fidelle M, Rizvi H, et al. Negative association of antibiotics on clinical activity of immune checkpoint inhibitors in patients with advanced renal cell and non-small-cell lung cancer. Ann Oncol 2018;29:1437-44.

18. Pflug N, Kluth S, Vehreschild JJ, Bahlo J, Tacke D, Biehl L, et al. Efficacy of antineoplastic treatment is associated with the use of antibiotics that modulate intestinal microbiota. OncoImmunology 2016;5:e1150399.

19. Hammes WP, Neuhaus FC. On the mechanism of action of vancomycin: inhibition of peptidoglycan synthesis in Gaffkya homari. Antimicrob Agents Chemother 1974;6:722-8.

20. Demirci H, Murphy F, Murphy E, Gregory ST, Dahlberg AE, Jogl G. A structural basis for streptomycin-induced misreading of the genetic code. Nat Commun 2013;4:1355. doi: 10.1038/ncomms2346.: 1355 .

21. Ponzetta A, Carriero R, Carnevale S, Barbagallo M, Molgora M, Perucchini C, et al. Neutrophils Driving Unconventional T Cells Mediate Resistance against Murine Sarcomas and Selected Human Tumors. Cell 2019;178:346-60.

22. Jaime-Ramirez AC, Mundy-Bosse BL FAU - Kondadasula S, Kondadasula S FAU Jones N, Jones NB FAU - Roda J, Roda JM FAU - Mani A, Mani AF, et al. IL-12 enhances the antitumor actions of trastuzumab via NK cell IFN-+1 production. J Immunol 2011;186:3401-9.

23. Rakoff-Nahoum S, Paglino J, Eslami-Varzaneh F, Edberg S, Medzhitov R. Recognition of commensal microflora by toll-like receptors is required for intestinal homeostasis. Cell 2004;118:229-41.

24. Rodrigues RR, Greer RL, Dong X, DSouza KN, Gurung M, Wu JY, et al. AntibioticInduced Alterations in Gut Microbiota Are Associated with Changes in Glucose Metabolism in Healthy Mice. Front Microbiol 2017;8:2306. 
25. Caporaso JG, Kuczynski J, Stombaugh J, Bittinger K, Bushman FD, Costello EK, et al. QIIME allows analysis of high-throughput community sequencing data. Nat Methods 2010;7:335-6.

26. Prat A, Bianchini G, Thomas M, Belousov A, Cheang MC, Koehler A, et al. Researchbased PAM50 subtype predictor identifies higher responses and improved survival outcomes in HER2-positive breast cancer in the NOAH study. Clin Cancer Res 2014;20:511-21 doi: 10.1158/1078-0432.CCR-13-0239.

27. Huang DW, Sherman BT, Lempicki RA. Systematic and integrative analysis of large gene lists using DAVID bioinformatics resources. Nat Protoc 2009;4:44-57.

28. Subramanian A, Tamayo P, Mootha VK, Mukherjee S, Ebert BL, Gillette MA, et al. Gene set enrichment analysis: a knowledge-based approach for interpreting genome-wide expression profiles. Proc Natl Acad Sci U S A 2005;102:15545-50.

29. Triulzi T, Casalini P, Sandri M, Ratti F, Carcangiu ML, Colombo MP, et al. Neoplastic and stromal cells contribute to an extracellular matrix gene expression profile defining a breast cancer subtype likely to progress. PLoS ONE 2013;8:e56761.

30. Rody A, Holtrich U, Pusztai L, Liedtke C, Gaetje R, Ruckhaeberle E, et al. T-cell metagene predicts a favorable prognosis in estrogen receptor-negative and HER2-positive breast cancers. Breast Cancer Res 2009;11:R15.

31. Charoentong P, Finotello F, Angelova M, Mayer C, Efremova M, Rieder D, et al. Pancancer Immunogenomic Analyses Reveal Genotype-Immunophenotype Relationships and Predictors of Response to Checkpoint Blockade. Cell Rep 2017;18:248-62.

32. Segata N, Izard J, Waldron L, Gevers D, Miropolsky L, Garrett WS, et al. Metagenomic biomarker discovery and explanation. Genome Biol 2011;12:R60-12.

33. Lozupone C, Knight R. UniFrac: a new phylogenetic method for comparing microbial communities. Appl Environ Microbiol 2005;71:8228-35.

34. Warton DI, Wright ST, Wang I. Distance-based multivariate analyses confound location and dispersion effects. Methods in Ecology and Evolution 2012;3:89-101.

35. Castagnoli L, Iezzi M, Ghedini GC, Ciravolo V, Marzano G, Lamolinara A, et al. Activated d16HER2 homodimers and Src kinase mediate optimal efficacy for trastuzumab. Cancer Res 2014;74:6248-59.

36. Stagg J, Loi S, Divisekera U, Ngiow SF, Duret H, Yagita H, et al. Anti-ErbB-2 mAb therapy requires type I and II interferons and synergizes with anti-PD-1 or anti-CD137 mAb therapy. Proc Natl Acad Sci USA 2011;108:7142-7 doi: 10.1073/pnas.1016569108.

37. Park S, Jiang Z, Mortenson ED, Deng L, Radkevich-Brown O, Yang X, et al. The therapeutic effect of anti-HER2/neu antibody depends on both innate and adaptive immunity. Cancer Cell 2010;18:160-70 doi: 10.1016/j.ccr.2010.06.014. 
38. Parada Venegas D, De la Fuente MK, Landskron G, Gonz+ílez MJ, Quera R, Dijkstra G, et al. Corrigendum: Short Chain Fatty Acids (SCFAs)-Mediated Gut Epithelial and Immune Regulation and Its Relevance for Inflammatory Bowel Diseases. Front Immunol 2019;10:1486.

39. Rossi T, Vergara D, Fanini F, Maffia M, Bravaccini S, Pirini F. Microbiota-Derived Metabolites in Tumor Progression and Metastasis. Int J Mol Sci 2020;21.

40. Mohsin SK, Weiss HL, Gutierrez MC, Chamness GC, Schiff R, DiGiovanna MP, et al. Neoadjuvant trastuzumab induces apoptosis in primary breast cancers. J Clin Oncol 2005;23:2460-8.

41. Triulzi T, Regondi V, De Cecco L, Cappelletti MR, Di Modica M, Paolini B, et al. Early immune modulation by single agent trastuzumab as a marker of trastuzumab benefit. $\mathrm{Br} \mathbf{J}$ Cancer 2018;119:1487-94.

42. Koyama M, Mukhopadhyay P, Schuster IS, Henden AS, Hulsdunker J, Varelias A, et al. MHC Class II Antigen Presentation by the Intestinal Epithelium Initiates Graft-versusHost Disease and Is Influenced by the Microbiota. Immunity 2019;51:885-98.

43. Schaupp L, Muth S, Rogell L, Kofoed-Branzk M, Melchior F, Lienenklaus S, et al. Microbiota-Induced Type I Interferons Instruct a Poised Basal State of Dendritic Cells. Cell 2020;181:1080-96.

44. Vignali DA, Kuchroo VK. IL-12 family cytokines: immunological playmakers. Nat Immunol 2012;13:722-8.

45. Mortenson ED, Park S, Jiang Z, Wang S, Fu YX. Effective anti-neu-initiated antitumor responses require the complex role of CD4+ T cells. Clin Cancer Res 2013;19:1476-86 doi: 10.1158/1078-0432.CCR-12-2522.

46. Datta J, Berk E, Xu S, Fitzpatrick E, Rosemblit C, Lowenfeld L, et al. Anti-HER2 CD4(+) T-helper type 1 response is a novel immune correlate to pathologic response following neoadjuvant therapy in HER2-positive breast cancer. Breast Cancer Res 2015;17:71.

47. Scott NA, Andrusaite A, Andersen P, Lawson M, Alcon-Giner C, Leclaire C, et al. Antibiotics induce sustained dysregulation of intestinal $\mathrm{T}$ cell immunity by perturbing macrophage homeostasis. LID - 10.1126/scitranslmed.aao4755 [doi] LID - eaao4755. Sci Transl Med 2018;10:eaao4755.

48. Varadan V, Gilmore H, Miskimen KL, Tuck D, Parsai S, Awadallah A, et al. Immune signatures following single dose trastuzumab predict pathologic response to preoperative trastuzumab and chemotherapy in HER2-positive early breast cancer. Clin Cancer Res 2016;22:3249-59.

49. Spencer CN, Gopalakrishnan V, McQuade J, Andrews MC, Helmink B, Wadud Khan MA, et al. The gut microbiome (GM) and immunotherapy response are influenced by 
Author Manuscript Published OnlineFirst on January 22, 2021; DOI: 10.1158/0008-5472.CAN-20-1659

Author manuscripts have been peer reviewed and accepted for publication but have not yet been edited.

644

645

host lifestyle factors. Proceedings: AACR Annual Meeting 2019 March 29-April 3, 2019; Atlanta, GA, -DOI: 10.1158/1538-7445.AM2019-2838. 2019.

50. Gingras I, Gebhart G, De Azambuja E, Piccart-Gebhart M. HER2-positive breast cancer is lost in translation: time for patient-centered research. Nat Rev Clin Oncol 2017;14:66981. 
Table 1. Clinical characteristics of the patients analyzed in this study.

647

\begin{tabular}{|c|c|c|c|}
\hline & $R, n=16$ & $\mathbf{N R}, \mathbf{n}=\mathbf{8}$ & $p$-value* \\
\hline Age (years) & & & $0.155^{\ddagger}$ \\
\hline Median (range) & $54(36-80)$ & $61(41-76)$ & \\
\hline Tumor size $^{C^{c}}$ & & & 1.00 \\
\hline$\leq 2 \mathrm{~cm}$ & $6(43 \%)$ & $4(50 \%)$ & \\
\hline$>2 \mathrm{~cm}$ & $8(57 \%)$ & $4(50 \%)$ & \\
\hline Lymph node status & & & 0.064 \\
\hline Negative & $3(19 \%)$ & $5(63 \%)$ & \\
\hline Positive & $13(81 \%)$ & $3(37 \%)$ & \\
\hline ER status & & & 0.657 \\
\hline Negative & $7(44 \%)$ & $2(25 \%)$ & \\
\hline Positive & $9(56 \%)$ & $6(75 \%)$ & \\
\hline PGR status & & & 0.189 \\
\hline Negative & $7(44 \%)$ & $1(12 \%)$ & \\
\hline Positive & $9(56 \%)$ & $7(88 \%)$ & \\
\hline Grade & & & 1.00 \\
\hline I and II & $8(50 \%)$ & $4(50 \%)$ & \\
\hline III & $8(50 \%)$ & $4(50 \%)$ & \\
\hline
\end{tabular}

648 ER, estrogen receptor; PGR, progesterone receptor; R, pathological complete response; NR, non-responsive; ${ }^{6} \mathrm{R}$, $649 \mathrm{n}=14$ and NR, $n=8$. $^{*}$, p-value of Fisher's exact test. ${ }^{\neq}$, p-value of the Mann-Whitney test 